\title{
Multimode MEMS Resonator for Simultaneous Sensing of Vapor Concentration and Temperature
}

\author{
N. Jaber, S. Ilyas, O. Shekhah, M. Eddaoudi, and M. I. Younis
}

\begin{abstract}
Most gas sensors suffer from the cross sensitivity to environmental temperature, which significantly reduces the accuracy and reliability of measurements. Current solutions require the fabrication of a thermometer in close proximity to the gas sensor or an identical reference sensor to compensate for the sensor drift due to temperature. This increases the device size, fabrication cost, and the power required to operate the sensor; and also adds to the complexity of the device circuit for signal processing. Here, we demonstrate a single resonant gas sensor, based on a microbeam uniformly coated with metal-organic frameworks (MOFs), capable of simultaneously measuring environmental temperature and gas concentration (water vapor). Using the electrostatic harmonic voltage, we actuate the microbeam simultaneously near the first and second vibration modes. The frequency shifts of these two modes due to physical stimuli changes are monitored in real time. The lower electrode of the clamped-clamped microbeam resonator is perforated to reduce the effect of squeeze film damping, thereby allowing operation under atmospheric pressure. We demonstrate experimentally the effectiveness of this technique to measure the environmental temperature and gas concentration. Based on the theoretical analysis and the Allan deviation results, a minimum detectable temperature of $0.03{ }^{\circ} \mathrm{C}$ and water vapor concentration of 4.6 ppm is demonstrated.
\end{abstract}

Index Terms - Multimodal, MOFs coating, electrostatic actuation, humidity and temperature sensing, and resonators.

\section{INTRODUCTION}

$\mathrm{D}$ eveloping a smart sensor is of crucial importance in health and environmental monitoring [1], biological and gas hazards detection and warnings [2], aerospace systems [3], and biological application [4]. Commonly, intelligent sensors are composed of multiple sensing elements to measure the different physical stimuli and a microcontroller to process the signal and provide useful information for the user or perform certain actions [5]. Electrostatically actuated MEMS based resonators have been the main building block of many biological, chemical, and gas sensors. This can be attributed to the unique advantages of low power consumption, ease of

(Corresponding author: Mohammad I. Younis.)

The authors are with the King Abdullah University of Science and Technology, Thuwal 23955-6900, Saudi Arabia (e-mail: nizar.jaber@kaust.edu.sa; saad.ilyas@kaust.edu.sa; Osama.Shekhah@kaust .edu.sa; mohamed.eddaoudi@kaust.edu.sa; mohammad.younis@kaust.edu .sa). fabrication, and compatibility with CMOS circuits [6]. The operation principle is based on tracking the frequency shift in the fundamental mode due to external stimuli, such as gas adsorption [7], pressure change [8], and protein detection [9]. However, the need to operate them under controlled environmental conditions and at low pressure limited their practicality [10]. Furthermore, the low-quality factor in atmospheric pressure hinders their sensitivity for gas and mass sensing applications.

In a previous work, we presented an optimized resonator design to achieve an acceptable quality factor allowing operation in air. Also, we enhanced the excitation of the higher order modes of vibration, of higher quality factor, which resulted in higher sensitivity for gas sensing [11].

Recently, significant attention has been dedicated to exploit information from the different modes of vibration of a MEMS resonator for various sensing applications, such as to extract the mechanical properties and mass of the adsorbed analyte [12], quantify the amount of attached beads or proteins on the resonator surface and locate their positions [9], weigh nanoparticles flowing inside a microfluidic channel while tracking their positions and velocities [13], and detect phase change from solid to liquid of Polyethylene Glycol [14]. Also, the coupling of two different modes has been utilized to enhance the stability and reduce fluctuation of MEMS resonators [15].

Selective detection of gases is achieved by functionalizing the resonator surface with thin films that have affinity for a particular type of gases. Depending on the type of gas, different materials have been engineered and synthesized, such as gold for mercury detection [16], zeolitic imidazolate framework (ZIF) for $\mathrm{CO}_{2}$ detection [17], polymer dispersed liquid crystals doped with carbon nanotubes for Acetone detection [18], and palladium coating for hydrogen sensing [19]. Metal-organic frameworks (MOFs) are new evolving class of porous materials that are composed of metal nodes connected with organic links. These materials have gained significant attentions due to the large surface area, low density, tunable pore size, and exposed inner functionalities [20- 24]. Depending on the type of metal atoms and the organic links, different types of MOFs have been synthesized to selectively detect $\mathrm{H}_{2} \mathrm{~S}$ [25], $\mathrm{CO}_{2}$ [26], volatile organic compounds (VOCs) [7], and humidity [27].

In gas sensing, the effect of environmental disturbances and the cross-sensitivity between the temperature and the amount of adsorbed gas on the sensor surface dramatically reduce the accuracy and reliability of measurements [28-30]. Several 
attempts have been reported in the literature to address this issue by fabricating an additional thermometer and a reference sensor in close proximity to the gas sensor. The signal from the gas sensor is corrected based on the thermometer reading [31]. Another technique is by functionalizing two identical devices with different films that have different response coefficients to temperature and humidity changes. The signals from the two devices can be utilized to quantify the variation in these physical parameters [32]. However, the need for additional physical sensors and circuits for signal postprocessing raises the fabrication cost, increases the device footprint, and adds extra demand on the power required to operate these sensors.

Thus, there is a need to develop sensitive sensors that can simultaneously sense multiple physical stimuli with the minimum power consumption. In this paper, we utilize two modes of vibrations of an electrostatically actuated MEMS resonator functionalized with MOFs, and operated in air, to sense the change in two physical stimuli. As a case study, we demonstrate this concept to detect the temperature change and water vapor concentration. However, this concept can be extended to sense other physical parameters, such as pressure and other gas types, by optimizing the structure design to be able to actuate and track higher number of modes.

\section{FABRICATION}

The proposed sensor is composed of an electrostatically actuated clamped-clamped microbeam, Fig. 1(a), excited using a half electrode configuration to enhance the excitation of the second mode of vibration. The sensor is fabricated on a 4" silicon wafer coated with $3 \mu \mathrm{m}$ of silicon dioxide $\left(\mathrm{SiO}_{2}\right)$ layer. The $\mathrm{SiO}_{2}$ layer is deposited using the plasma enhanced physical-chemical vapor deposition (PECVD) to provide the electrical insulation between the device and the wafer. Using the PECVD tool, a $7 \mu \mathrm{m}$ thick amorphous silicon $(\alpha-\mathrm{Si})$ is deposited and then polished to a final thickness of $\sim 3.5 \mu \mathrm{m}$. The polishing is conducted to remove the concavities in the surface due to the etching holes in the $\mathrm{SiO}_{2}$ layer (underneath layer). The $\alpha$-Si acts as a sacrificial layer that defines the air gap between the two electrodes and will be etched during the final release step. The microbeam is composed of a $4.3 \mu \mathrm{m}$ polyimide coated from the bottom with $\mathrm{Cr} / \mathrm{Au} / \mathrm{Cr}$ layer of thicknesses 50/200/50 $\mathrm{nm}$, which forms the upper electrode of the resonator. The microbeam is coated from the top with $\mathrm{Cr} / \mathrm{Au}$ layer of thickness 50/200 $\mathrm{nm}$, which acts as a hard mask to protect the microbeam during the etching process of the polyimide. This layer is functionalized with a $\mathrm{COOH}-$ terminated layer by immersing the chip in ethanolic solution of 16-mercaptohexadecanoic acid for 24 hours [33, 34]. Using the layer-by-layer approach, a uniform MOF layer is grown by dipping the chip in copper acetate metal precursor ethanolic solution and organic ligand (terephthalic acid (bcd)) ethanolic solution for five and ten minutes, respectively, to grow the $\mathrm{Cu}$ (bcd).xH2O MOF thin film [35]. The process is repeated for ten cycles where the chip is rinsed with ethanol every cycle [32]. In previous study [11], we studied the cross sensitivity of the utilized type of MOFs with other gases. Our results showed a strong affinity to water vapor compared with Acetone and ethanol. To release the microbeam, the sacrificial $\alpha$-Si layer is fully etched using the $\mathrm{XeF}_{2}$ dry etchant. Extra etching cycles where conducted to etch the silicon from the opening in the $\mathrm{SiO}_{2}$ layer and form the perforations in the lower electrode. Figure 1a and $1 \mathrm{~b}$ show a schematic of the microbeam design and the fabrication steps. Figure 1c shows an SEM image and a schematic of the clamped-clamped resonator; of length (l) $400 \mu \mathrm{m}$, width (w) $50 \mu \mathrm{m}$, and gap (g) $3.3 \mu \mathrm{m}$. The formation of the MOF thin film was confirmed using X-ray diffraction (XRD) as depicted in Fig. 1d, which shows that crystalline thin film from the 10 cycles growth was obtained. The peak at 37.5 corresponds to the Gold layer that forms the connections pad. The lower electrode was perforated with a perforation diameter $d=20 \mu \mathrm{m}$, and with spacing between holes $S=15 \mu \mathrm{m}$. These dimensions were selected to minimize squeeze film damping in accordance with the fabrication process rules [36]. The insets of Fig. 1a shows the uniformity of the coating over the microbeam surface.

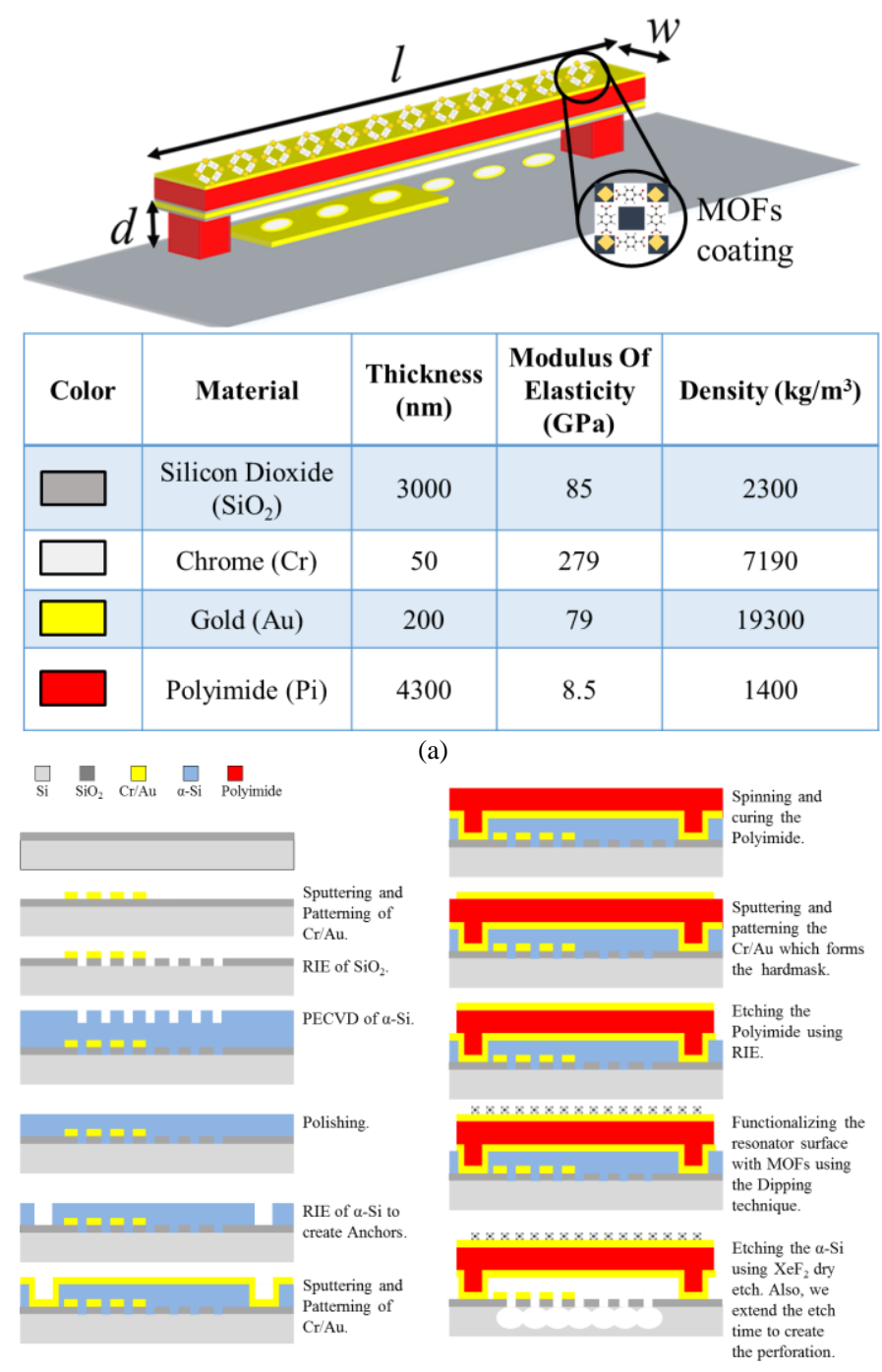

(b) 


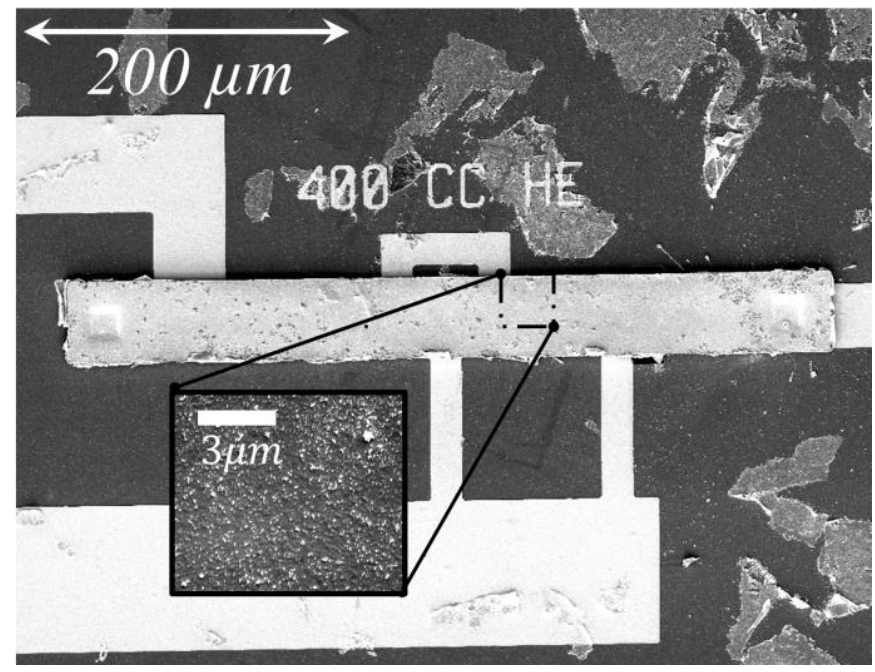

(c)

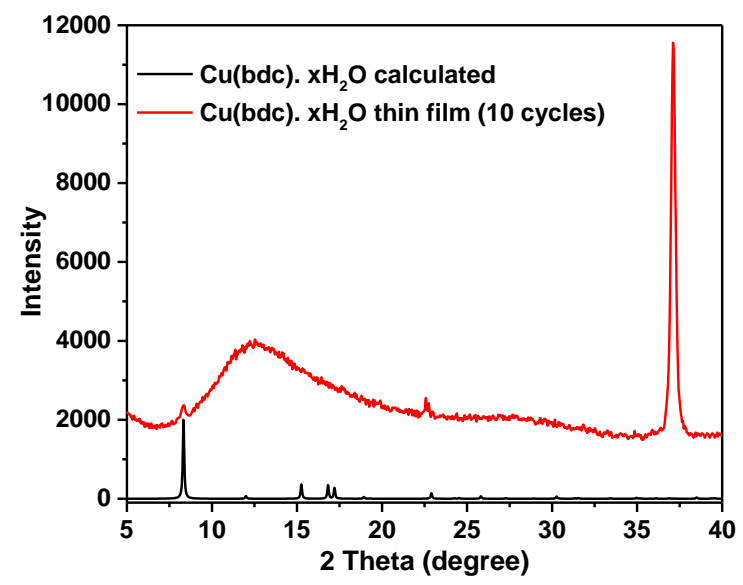

(d)

Fig. 1. (a) Schematic of the microbeam with the lower electrode perforation showing the material layers types, properties, and thicknesses. (b) Schematic of the fabrication process steps. (c) An SEM image of the fabricated microbeam with MOFs functionalization. (d) $\mathrm{XRD}$ of $\mathrm{Cu}(\mathrm{bdc}) \cdot \mathrm{xH}_{2} \mathrm{O} \mathrm{MOF}$ thin film grown on the microbeam (red) and its calculated pattern (black).

\section{CHARACTERIZATION}

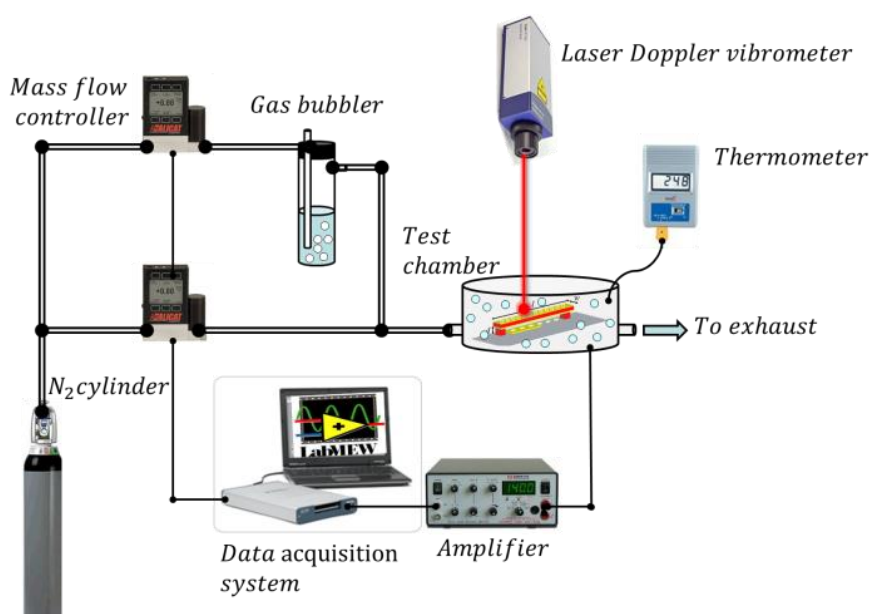

Fig. 2. Schematic of the experimental setup used for testing the device.

To characterize the microbeam, we use a Laser Doppler vibrometer to monitor the response, reveal the frequencies, mode shapes of vibration, and track the change in the amplitude of vibration due to gas exposure and temperature change. We utilize a data acquisition card (DAQ) to actuate the microbeam with different types of $\mathrm{AC}$ signals superimposed to a DC voltage. Also, the DAQ is used to acquire the microbeam response from the laser. Using the LabVIEW software, the recorded real time data are postprocessed to generate the frequency response curves. Also, we record the shift in the amplitude of vibration at the operating frequencies due to physical stimuli change. All measurements are conducted by focusing the laser point at quarter of the microbeam length. By employing the frequency response curves, the recorded amplitude shift is converted into frequency values. The microbeam is placed into the test chamber, which is equipped with ports to provide the actuation signals, a photothermal heater to regulate the chamber temperature, a thermometer, a pressure gauge, and is opened to the atmospheric pressure, as shown in Fig. 2. The water vapor is generated by flowing the nitrogen inside a bubbler placed in a controlled temperature bath. The vapor concentration is governed by controlling the flow of the dry nitrogen line and bubbler line. The experimental setup is depicted in Fig. 2.

\section{MATHEMATICAL MODEL}

To demonstrate the concept of simultaneously sensing the change in water vapor concentration and environmental temperature, we consider the governing equation of motion of an electrostatically actuated clamped-clamped microbeam of length $l$, width $b$, and thickness $h$. The mass $m$ of the adsorbed gas on the resonator surface is assumed to be uniformly distributed over the microbeam length. A change in the chamber temperature $\Delta T$ induces an axial stress along the microbeam axis. The equation of motion can be written as [6]

$$
\begin{aligned}
& \frac{(M+m)}{l} \frac{\partial^{2} W}{\partial t^{2}}+E I \frac{\partial^{4} W}{\partial x^{4}}+c \frac{\partial W}{\partial t}= \\
& \frac{\varepsilon b\left(V_{D C}+V_{A C 1} \operatorname{Cos}\left(\Omega_{1} \mathrm{t}\right)+V_{A C 2} \operatorname{Cos}\left(\Omega_{2} \mathrm{t}\right)\right)^{2}}{2(g-W)^{2}} \\
& +\left(N-\alpha \Delta T+\frac{E b h}{2 l} \int_{0}^{l}\left(\frac{\partial W}{\partial x}\right)^{2} d x\right) \frac{\partial^{2} W}{\partial x^{2}}
\end{aligned}
$$

where $M$ is the microbeam mass, $d$ is the air gap width between the two electrodes, $E I$ is the flexural of rigidity, $c$ is the viscous damping, $\varepsilon$ is the air permittivity, $\alpha$ is the thermal expansion coefficient, $N$ is the axial force due to the residual stress from the fabrication, and $W$ is the out of plane deflection. The electrostatic force is composed of two AC sources $V_{A C l}$ and $V_{A C 2}$ of frequencies $\Omega_{1}$ and $\Omega_{2}$, respectively, superimposed to a DC load $V_{D C}$. The boundary conditions of 


$$
\begin{array}{ll}
\text { the } & \text { clamped-clamped } \\
w(0, t)=0 & \frac{\partial w}{\partial x}(0, t)=0 \\
w(l, t)=0 & \frac{\partial w}{\partial x}(l, t)=0
\end{array}
$$

microbeam

are

The corresponding unforced and undamped eigenvalue problem of Eq. (1) is written as

$$
\phi_{m}^{\prime \prime \prime \prime}(x)-\left(N_{n o n}-\alpha_{n o n} \Delta T\right) \phi_{m}^{\prime \prime}(x)-\left(1+\frac{m}{M}\right) \omega_{m}^{2} \phi_{m}(x)=0
$$

where $N_{n o n}=N l^{2} / E I$ is the nondimensional axial stress, $\alpha_{n o n}=\alpha l^{2} / E I$ is the nondimesional thermal expansion coefficient, $\omega_{n o n_{m}}=\sqrt{m l^{3} / E I} \omega_{m}$ is the $m^{\text {th }}$ mode nondimensional eigenfrequency, $\omega_{m}$ is the $m^{\text {th }}$ mode dimensional natural frequency, $\phi_{m}(x)$ is the $m^{\text {th }}$ unforced, undamped, and orthonormal clamped-clamped microbeam mode shape, and the prime refers to the spatial derivative. To obtain an analytical expression for the frequency shift due to a temperature change $\Delta T$ and an adsorbed mass $m$, we solve the eigenvalue problem Eq. (3) using a one-mode Galerkin procedure [37]. By multiplying Eq. (3) with the $m^{\text {th }}$ mode shape and integrating along the beam length we obtain

$$
\omega_{m}=\sqrt{\frac{\int_{0}^{1} \phi_{m}^{(4)}(x) \times \phi_{m}(x)-\left(N_{n o n}-\alpha_{n o n} \Delta T\right) \int_{0}^{1} \phi_{1}^{(2)}(x) \times \phi_{1}(x) d x}{(1+m / M)}}
$$

The parameters $N_{n o n}$ and $\alpha_{n o n}$ can be extracted experimentally by following the procedure demonstrated in the Appendix. Hence, the only unknowns in Eq. (4) are the chamber temperature and the amount of mass $m$ adsorbed on the resonator surface. Theoretically, tracking the frequency shift of two modes of vibration is enough to quantify the change in these two physical parameters. In our experiments, the $1^{\text {st }}$ and $2^{\text {nd }}$ mode of vibration are utilized as follows

$$
\omega_{1}=\sqrt{\frac{\int_{0}^{1} \phi_{1}^{(4)}(x) \times \phi_{1}(x) d x-\left(N_{n o n}-\alpha_{n o n} \Delta T\right) \int_{0}^{1} \phi_{1}^{(2)}(x) \times \phi_{1}(x) d x}{(1+m / M)}}
$$

$$
\omega_{2}=\sqrt{\frac{\int_{0}^{1} \phi_{2}^{(4)}(x) \times \phi_{2}(x) d x-\left(N_{n o n}-\alpha_{n o n} \Delta T\right) \int_{0}^{1} \phi_{2}^{(2)}(x) \times \phi_{2}(x) d x}{(1+m / M)}}
$$

(6)

From Eq. (5) and Eq. (6), we can find out the temperature change and amount of adsorbed mass. However, in gas sensing, the amount of gas adsorbed on the resonator surface depends on the microbeam temperature [28-30], which implies that Eq. (5) and Eq. (6) may not be sufficient to extract the unknown parameters. To eliminate the effect of added mass, we divide Eq. (5) and Eq. (6) to obtain the ratio equation, which depends only on temperature change as below

$$
R=\frac{\omega_{2}}{\omega_{1}}=\sqrt{\frac{\int_{0}^{1} \phi_{2}^{(4)}(x) \times \phi_{2}(x) d x-\left(N_{n o n}-\alpha_{n o n} \Delta T\right) \int_{0}^{1} \phi_{2}^{(2)}(x) \times \phi_{2}(x) d x}{\int_{0}^{1} \phi_{1}^{(4)}(x) \times \phi_{1}(x) d x-\left(N_{n o n}-\alpha_{n o n} \Delta T\right) \int_{0}^{1} \phi_{1}^{(2)}(x) \times \phi_{1}(x) d x}}
$$

In Figure S3 (appendix material), we plot Eq. (5), Eq. (6), and Eq. (7) for various values of temperature and added mass. Equation (7) and Figure S3c show that the frequency ratio $R$ depends only on the temperature change and is independent of the amount of mass being adsorbed on the resonator surface. Using this equation, the temperature change values can be extracted based on the measured frequency values. By substituting back into Eq. (5) or Eq. (6), the amount of adsorbed mass can be found.

\section{FREQUENCY RESPONSE}

To experimentally extract the resonance frequency, we excite the microbeam with white noise signal and record the amplitude at different points along the microbeam length. During this test, the chamber pressure was fixed at 4 mTorr. We should mention here that although in the gas exposure experiments the beam will be actuated at atmospheric pressure, the extracted frequencies at the reduced pressure should not change much from their values at atmospheric pressure (potentially due to squeeze-film effect).

The measured resonance frequency values, Fig. $3 \mathrm{a}$, near the first $\left(f_{1}=117.3 \mathrm{kHz}\right)$ and second $\left(f_{2}=286.2 \mathrm{kHz}\right)$ mode of vibration are used to extract the residual axial force $N$ as shown in the appendix material. The recorded mode shapes, shown in the insets of Fig. 3a match the clamped-clamped microbeam mode shapes, which indicates the uniformity of the MOFs coating; nonuniform coating induces distortion in the mode shapes.

Next, we generate the frequency response curves by exciting the microbeam with a harmonic signal while recording the maximum amplitude $W_{\max }$ of vibration, which is for the second mode is at the quarter of the microbeam length. The excitation signal is composed of an AC signal $V_{A C}$ superimposed to a DC signal $V_{D C}$. Fig. $3 \mathrm{~b}$ and Fig. $3 \mathrm{c}$ show the results near the first mode and second mode of vibrations, respectively, for different $V_{A C}$ amplitudes and at atmospheric chamber pressure. These results are important to choose the suitable actuation voltage to operate the device with significant amplitudes above the noise level, and also for transforming the recorded amplitude shift due to gas exposure or temperature change into frequency data. The frequency shift values can be utilized in quantifying the amount of mass adsorbed on the resonator surface, and for extracting different thermodynamics and kinetics parameters that are essential for characterizing the coating material [38]. The microbeam response to a multifrequency signal is shown in Fig. 3d. The excitation signal is composed of two AC signals of frequencies: $\Omega_{l}=121 \mathrm{kHz}$, fixed near the first mode, and $\Omega_{2}$ which is swept near the second mode of vibration. The first source amplitude $V_{A C l}$ was varied as shown in Fig. 3d, while $V_{A C 2}$ was fixed at $V_{A C 2}=36 \mathrm{~V}$. These two signals were superimposed to a DC voltage $V_{D C}=50 \mathrm{~V}$. The reported frequency response curves, Fig. $3 \mathrm{~d}$, demonstrate the significance of multimode excitation in raising the floor of higher order modes above the noise level, which is important in sensing applications. Increasing the voltage amplitude near 
the first mode $V_{A C l}$ increases the floor of the second mode frequency response.

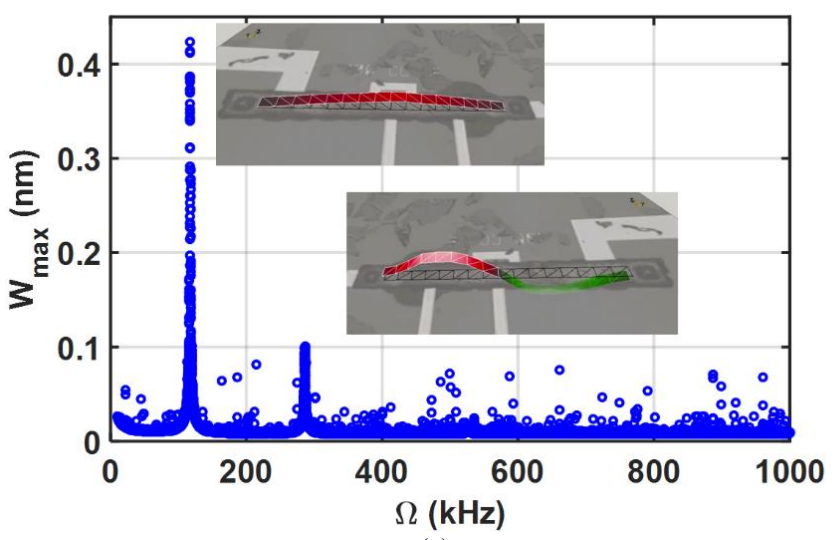

(a)

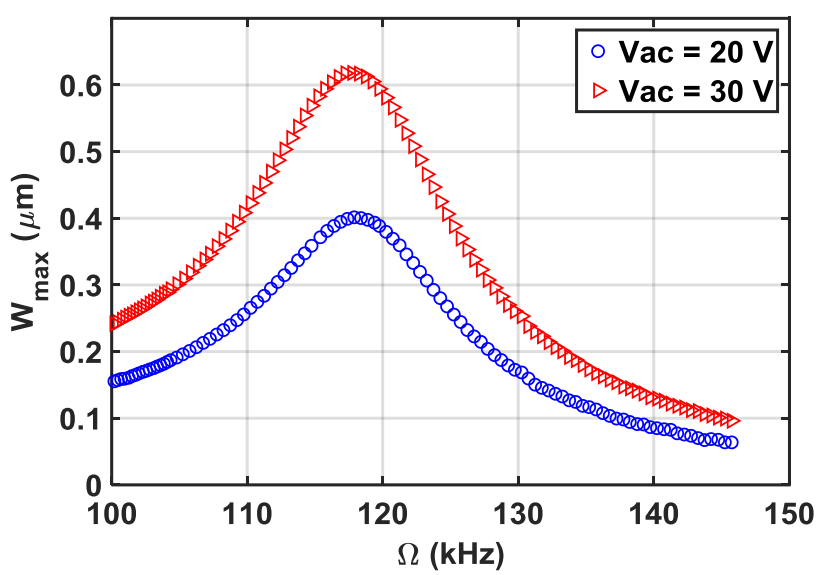

(b)

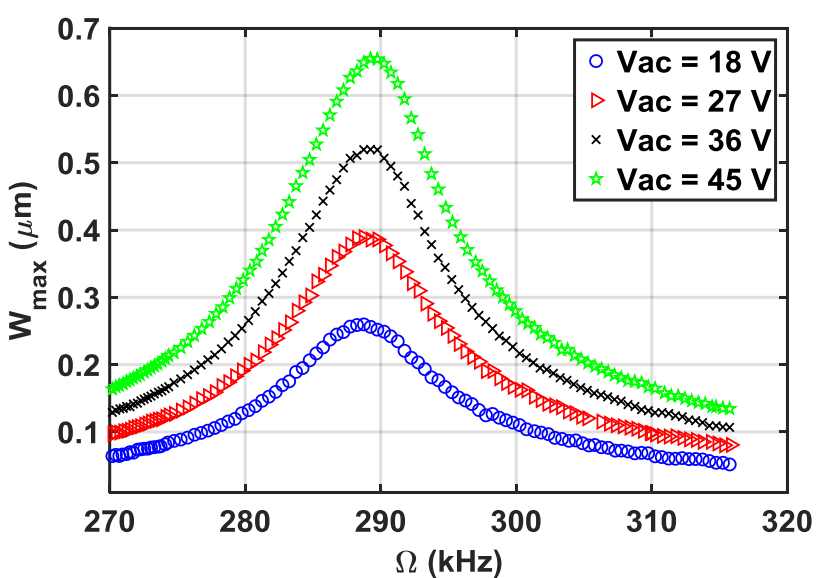

(c)

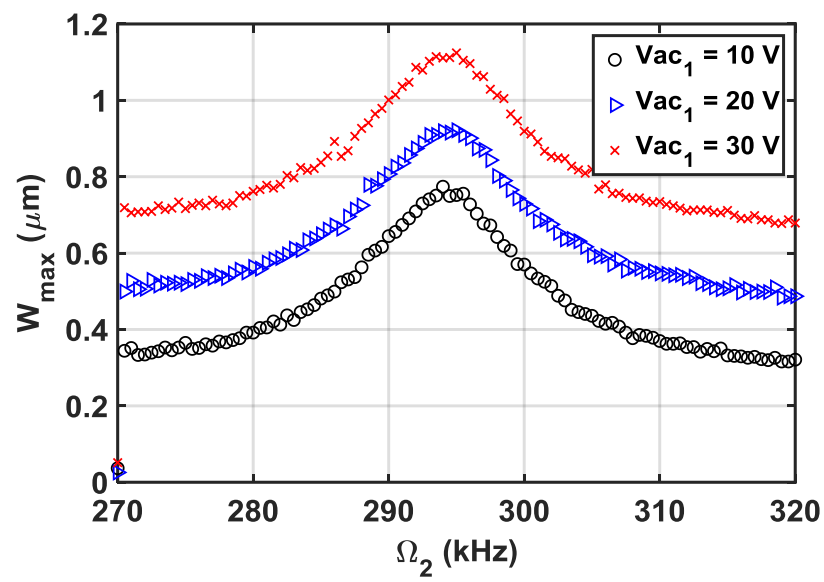

(d)

Fig. 3 Frequency response of the microbeam to (a) a white noise excitation at $V_{D C}=10 \mathrm{~V}, V_{A C}=15 \mathrm{~V}$, and at chamber pressure $P=4$ mTorr. Insets: the corresponding mode shapes acquired by recording the response at different point along the beam length. Frequency response of the microbeam at $V_{D C}=$ $50 \mathrm{~V}$ and at atmospheric chamber pressure for different AC voltages (b) near the first mode, (c) near the second mode. (d) Frequency response to a multifrequency excitation signal, $V_{A C l}$ as shown, $\Omega_{l}=121 \mathrm{kHz}, V_{A C 2}=36 \mathrm{~V}$, $\Omega_{2}=$ swept , and $V_{D C}=50 \mathrm{~V}$ at atmospheric pressure.

\section{NOISE ANALYSIS}

The sensor resolution can be evaluated by monitoring the amplitude evolution over time in the open loop configuration [39]. The resonator is actuated with harmonic signal composed of two frequencies $\Omega_{1}$ and $\Omega_{2}$ near the first and second modes of vibration, respectively. To record the amplitude fluctuation at each mode, we use LabVIEW software to perform a fast fourier transform FFT on the real time data of the microbeam response. The resulting frequency spectra is then used to record the amplitude at each frequency. Figure $4 \mathrm{a}$ shows a maximum amplitude fluctuation around $5 \mathrm{~nm}$ for the first mode and $10 \mathrm{~nm}$ for the second mode. This fluctuation can be attributed to intrinsic sources, such as defect motion [40], or extrinsic sources, such as thermo-mechanical noise, temperature fluctuation, adsorption-desorption noise, and instrumentation noise [41]. To further analyze the stability of the resonator, we calculate the Allan deviation. The amplitude fluctuation data, Fig. $4 \mathrm{a}$, is converted into frequency by utilizing the frequency response curves of the first and second mode depicted in Fig. 3b and Fig. 3c, respectively. The Allan deviation can be expressed as [38]

$\sigma_{f}(\tau)=\sqrt{\frac{1}{2(N-1)} \sum_{i=1}^{N-1}\left(\bar{f}_{i+1}^{\tau}-\bar{f}_{i}^{\tau}\right)^{2}}$

where $N$ is the sample size and $\bar{f}_{i}^{\tau}$ is the average frequency fluctuation over the $i^{\text {th }}$ time interval $\tau$ defined as

$\bar{f}_{i}(\tau)=\frac{f(i)-f_{0}}{f_{0}}$

where $f(i)$ is the measured frequency at the time step $i$, and $f_{0}$ is the resonator nominal frequency. The Allan deviation curve, depicted in Fig. 4b, shows a typical mechanical resonator behavior. For a short period of time the fluctuation is dominated by the white noise whereas for higher integration time the fluctuation increases due to random walk and steady 
drift [20]. The Allan deviation curves depicted in Fig. 4b show the enhanced stability of the second mode of vibration $\sigma_{f 2}(50)=18.67 \mathrm{ppm}$ compared with the first mode $\sigma_{f 1}(50)=39 \mathrm{ppm}$. The corresponding frequency fluctuation near the first mode and second mode were, respectively, $\Delta f_{1}=4.7 \mathrm{~Hz}$ and $\Delta f_{2}=5.5 \mathrm{~Hz}$.

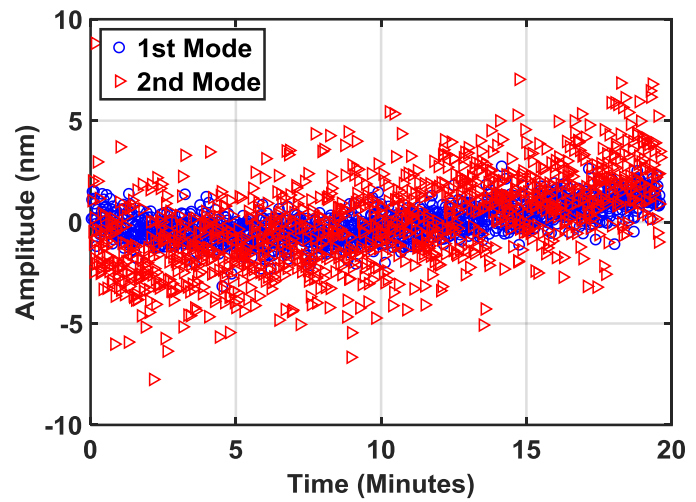

(a)

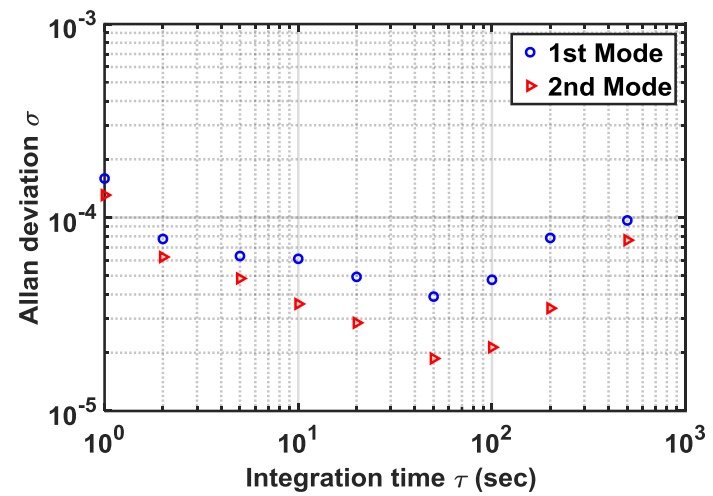

(b)

Fig. 4. (a) Amplitude fluctuation of the microbeam over 20 minutes to a multifrequency excitation signal $V_{A C l}=30 \mathrm{~V}, \Omega_{1}=121 \mathrm{kHz}, V_{A C 2}=36 \mathrm{~V}, \Omega_{2}$ $=294.5 \mathrm{kHz}$, and $V_{D C}=50 \mathrm{~V}$ at atmospheric pressure. (b) The corresponding Allan deviation near the first and second modes of vibration.

\section{OPERATING POINT SELECTION}

The vibration of the clamped-clamped microbeam induces a uniform strain across the beam generating axial stress that changes the beam stiffness. This can affect the frequency of the excited mode (self-tuning) or the frequency of the other modes (cross-tuning) [42, 43]. In the Euler-Bernoulli equation, Eq. (1), this effect is modeled as the mid-plane stretching term (the underlined term in Eq. (1)), which accounts for the beam elongation due to the out of plane vibration. To simplify the study, we select the operating frequencies such that the frequency shift due to the cross tuning is negligible. To experimentally find these frequencies, we actuate the microbeam with harmonic signals $\Omega_{l}$ and $\Omega_{2}$. $\Omega_{l}$ is fixed at $121 \mathrm{kHz}$ (first mode) while $\Omega_{2}$ is swept around the second mode of vibration. As shown in Fig. 5, the recorded dip in the first mode response is maximum near the second mode maximum amplitude $\left(\Omega_{2}=291 \mathrm{kHz}\right)$ due to the modes crosstuning. However, moving away from the second mode peak, the measured fluctuation in the first mode is below $5 \mathrm{~nm}$ and can be attributed to the external disturbances and noises as shown in the insets of Fig. 5. In our experiments, the second mode frequency was fixed at $294.5 \mathrm{kHz}$. Hence, as water vapor gets adsorbed and/or environmental temperature changes, the second mode frequency shifts to lower values and the amplitude at $294.5 \mathrm{kHz}$ falls down following the right branch of the second mode, which is the range with minimum cross-tuning effect as shown in the inset of Fig. 5.

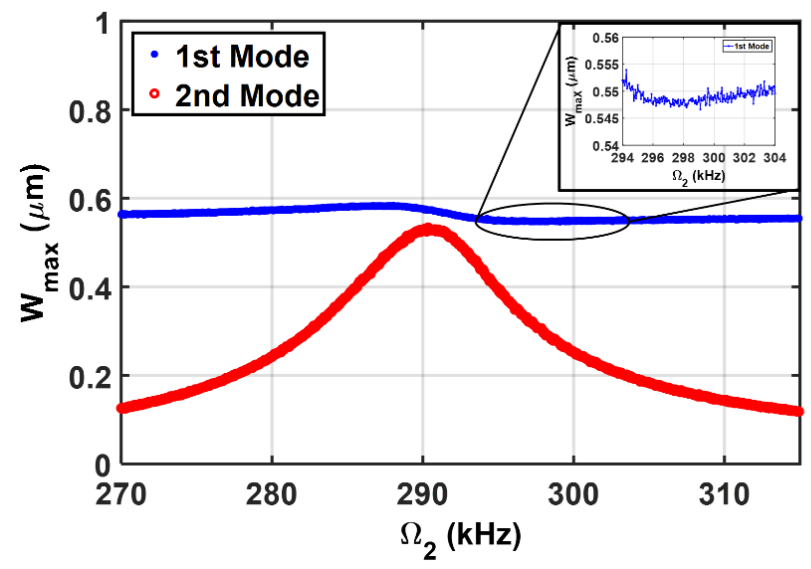

Fig. 5. The response of the microbeam to a fixed excitation near the first mode while sweeping the other source frequency near the second mode. $V_{A C l}=30 \mathrm{~V}$, $\Omega_{1}=121 \mathrm{kHz}, V_{A C 2}=36 \mathrm{~V}, \Omega_{2}=$ swept, and $V_{D C}=50 \mathrm{~V}$ at atmospheric pressure.

\section{EXPERIMENTAL RESULTS}

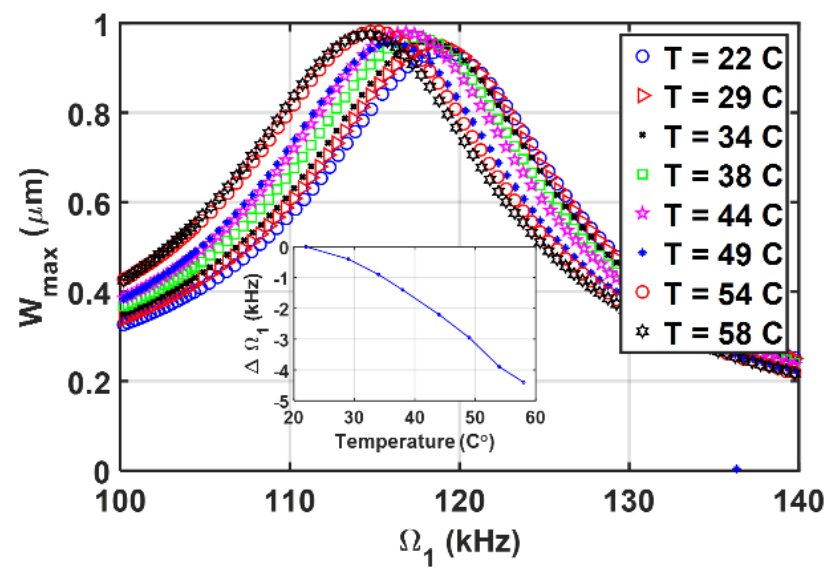

(a) 


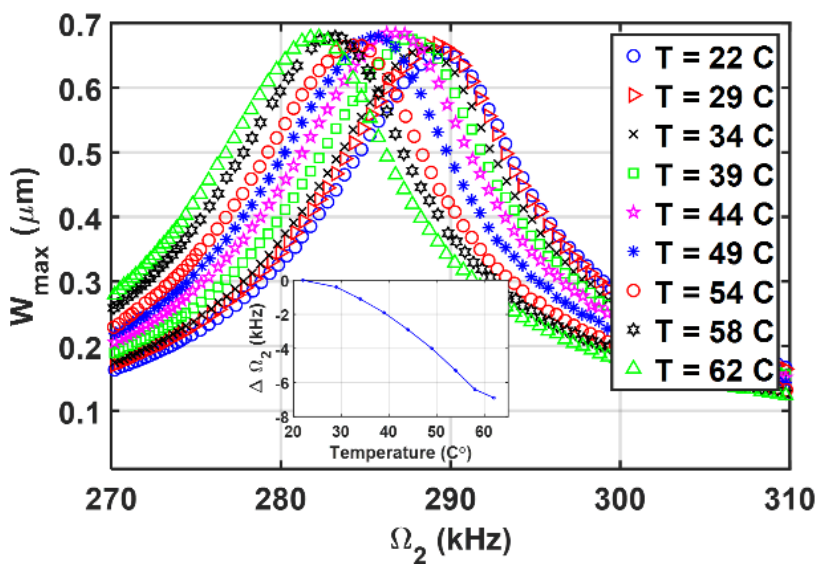

(b)

Fig. 6 Frequency response curves at $V_{D C}=50 \mathrm{~V}$, atmospheric pressure, and for different chamber temperature, (a) near the first mode at $V_{A C}=30 \mathrm{~V}$, (b) near the second mode at $V_{A C}=36 \mathrm{~V}$. Inset: the corresponding resonance frequency for each temperature value.

To experimentally extract the sensitivity of the resonator due to temperature change, we record the frequency response of the microbeam near the first and second modes at different chamber temperatures, Fig. 6. We utilized a photothermal heater to control the chamber temperature and a thermocouple placed in close proximity to the chip to record the temperature values. We fixed the photo thermal power at a particular value and waited for long time until the thermocouple reading reaches steady-state at fixed temperature value. Since the resolution of the used-thermometer is up to $1^{\circ} \mathrm{C}$, this implies potential inaccuracy in the temperature readings in the sub degree range.

The experimentally extracted responsivity near the first mode is $\mathfrak{R}_{T}^{1 s t}=128 \mathrm{~Hz} /{ }^{\circ} \mathrm{C}$ and for the second mode is $\mathfrak{R}_{T}^{2 n d}=187 \mathrm{~Hz} /{ }^{\circ} \mathrm{C}$, which indicates almost 1.5 times enhanced responsivity of the second mode as reported in the insets of Fig. 6. From the noise analysis, the minimum detectable temperature near the second mode is $\Delta T_{\min }=\Delta f_{\min } / \mathfrak{R}_{T}^{2 n d}=0.03^{\circ} \mathrm{C}$.

Figure 7 shows the real time measurements of the frequency shifts near the first and second modes of vibration at different chamber temperatures and water vapor concentrations. All the measurements were done inside the chamber at atmospheric pressure. Hence, changing the water vapor concentration did not affect the pressure or temperature. This was confirmed by monitoring the thermometer and the pressure gauge reading. The real time data were recorded after flushing the chamber with Nitrogen at $1 \mathrm{l} / \mathrm{min}$ flow for an extended period of time. The water vapor concentration $C$ is controlled by changing the nitrogen flow inside the bubbler. The temperature bath is fixed at $22^{\circ} \mathrm{C}$. The vapor concentration values are calculated following the procedure demonstrated in [11]. The chamber is flushed with $\mathrm{N}_{2}$ [19], to reduces the gas partial pressure, which helps in the desorption of the physisorbed gas molecules. As shown in Fig. 7a, Fig. 7b, and Fig. 7c, upon flushing the sensor with Nitrogen toward the end of the mass loading experiment, the resonator resonance frequency returns to its initial value, which confirms the reversibility of the sensor. The responsivity of the resonator due to water vapor adsorption at $T=22^{\circ} \mathrm{C}$ near the first and second modes are $\mathfrak{R}_{C}^{1 s t}=0.68 \mathrm{~Hz} / \mathrm{ppm}$ and $\mathfrak{R}_{C}^{2 n d}=1.2 \mathrm{~Hz} / \mathrm{ppm}$, respectively. The minimum detectable concentration is $\Delta C_{\min }=\Delta f_{\min } / \mathfrak{R}_{C}^{2 n d}=4.6 p p m$. As expected a better sensitivity is demonstrated near the second mode. The improved sensitivity near the second mode encourages the effort toward optimized resonator designs to efficiently excite the higher order modes of vibration for ultrasensitive applications.

By investigating the real time data shown in Fig. 7a, Fig. $7 \mathrm{~b}$, and Fig. 7c, we note that increasing the temperature at particular concentration values decreases the recorded frequency shift due to the reduced amount of water molecules adsorbed on the resonator surface. The same conclusion can be drawn from Fig. $7 b$ and Fig. 7c, where at higher concentration the frequency shifts at $22^{\circ} \mathrm{C}$ approach the measured values at $38^{\circ} \mathrm{C}$ for the first mode, and exceed them for the second mode.

We study the dynamics of absorption by fitting an exponential function $f(t)=\alpha \exp (-t / \tau)$ to the recorded experimental results at the different temperature values [17]. We utilize the recorded results near the second mode of vibration at $1762 \mathrm{ppm}$ to estimate the response time constant $\tau_{\text {Response }}$ and at $6431 \mathrm{ppm}$ to estimate the recovery time constant $\tau_{\text {Recovery. }}$ As shown in Table 1 , increasing the chamber temperature raises the response time constant and drops the recovery time. It is worth to mention that the current experimental setup limits the calculated response and recovery time. The chamber volume is $3 L$, and the maximum flow rate is $1.5 \mathrm{l} / \mathrm{min}$, which indicates that longer time is required to humidify and dehumidify the chamber.

Table 1. Response and recovery time constants based on the second mode real-time data at different temperature values.

\begin{tabular}{|c|c|c|}
\hline & $\begin{array}{c}\boldsymbol{\tau}_{\text {Response }}(\mathbf{m i n}) \text { at } \\
\mathbf{1 7 6 2} \mathbf{p p m}\end{array}$ & $\begin{array}{c}\boldsymbol{\tau}_{\text {Recovery }}(\mathbf{m i n}) \mathbf{a t} \\
\mathbf{6 4 3 1} \mathbf{~ p p m}\end{array}$ \\
\hline $\mathbf{T}=\mathbf{2 2}{ }^{\circ} \mathbf{C}$ & 4.65 & 27.15 \\
\hline $\mathbf{T}=\mathbf{3 8}^{\circ} \mathbf{C}$ & 7.5 & 18.92 \\
\hline $\mathbf{T}=\mathbf{4 5}^{\circ} \mathbf{C}$ & 7.85 & 15.59 \\
\hline
\end{tabular}

Theoretically, the frequency ratio $R$ should be horizontal straight lines for fixed chamber temperature and different water vapor concentrations as shown in Fig. S3c in the appendix. However, the experimentally recorded ratios show a slight deviation, which can be attributed to the temperature fluctuation (due the low resolution of the used thermocouple), and the different vibrational velocity of each point along the 
beam length. From basic adsorption theories, it is known that gas adsorption strongly depends on the surface temperature, gas concentration, and the velocity of the surface. Hence, even if the MOFs coating was uniformly distributed along the beam length, the difference in vibrational velocity along the beam results in position-dependent adsorption. To verify this, future experiments should be designed and conducted to quantify the velocity effect on adsorption.

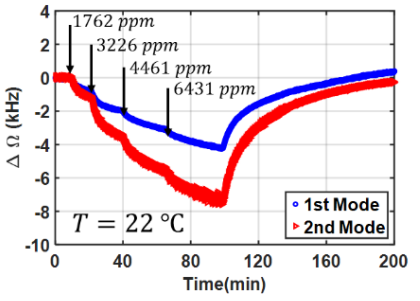

(a)

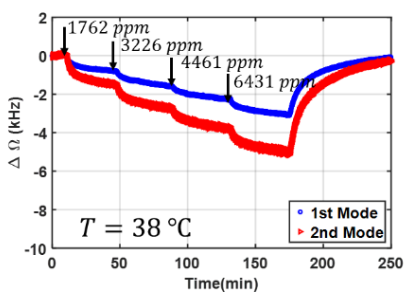

(b)

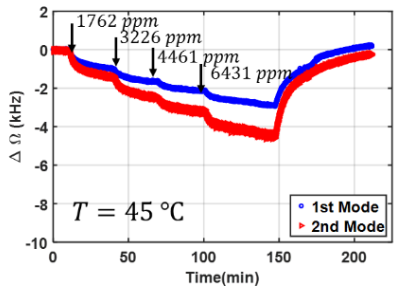

(c)

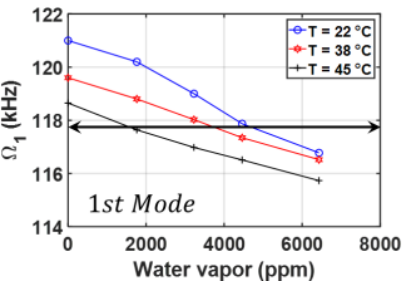

(d)

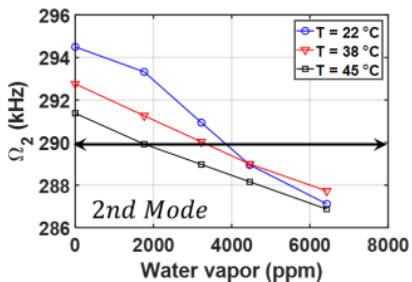

(e)

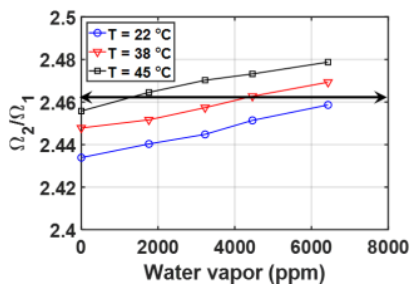

(f)

Fig. 7. Experimentally measured frequency shifts near the first and second mode of vibration for different water vapor concentrations at chamber temperature of (a) $22{ }^{\circ} \mathrm{C}$, (b) $38{ }^{\circ} \mathrm{C}$, and (c) $45{ }^{\circ} \mathrm{C}$. Summary of the experimentally measured frequency shifts at different combinations of water vapor concentration and chamber temperature near (d) the first mode, (e) the second mode, and (f) the two frequencies ratio.

The significance of the presented technique can be demonstrated by considering a case study where the experimentally measured frequency values near the first and second modes are $117.8 \mathrm{kHz}$ and $290 \mathrm{kHz}$, respectively. The corresponding frequency ratio $R$ is 2.461 . The case study data are highlighted with black arrow in Fig. 7d, Fig. 7e, and Fig. 7f. From the arrow intersection with the different curves, one can note that the only point that satisfies the measured frequency values and their corresponding ratio is $1750 \mathrm{ppm}$ concentration at $45^{\circ} \mathrm{C}$. Hence, using two modes of vibration of a single microbeam, it is possible to measure the change in two physical stimuli. These findings can be potentially extended to measure multiple physical parameters by increasing the number of modes such as the electrical charges.. It is worth to mention here that one needs to record the shift in the two resonance frequencies and the frequency ratio to determine the gas concentration and temperature uniquely. Also, the calibration steps have to be repeated for different sensors.

\section{CONCLUSIONS}

We presented a new technique to measure two physical stimuli using a single electrostatically actuated resonant microbeam coated with MOFs and operated in air. The method is based on simultaneously actuating and tracking two frequencies of two vibration modes of a microstructure. As a case study, we utilized this concept to measure the environmental temperature and humidity concentration. Based on the theoretical analysis and the Allan deviation results [44, 45], a minimum detectable temperature variation of $0.03{ }^{\circ} \mathrm{C}$ and a water vapor concentration of $4.6 \mathrm{ppm}$ were reported near the second mode of vibration. We presented a simple mathematical model to demonstrate the idea. Optimizing the resonator design to excite higher order modes will open the door for simultaneously measuring multiple physical stimuli using a single resonator, which reduces the power consumption, device size, and cost, and leads to smarter generation of sensors.

\section{ACKNOWLEDGEMENTS}

We acknowledge financial support from King Abdullah University of Science and Technology.

\section{REFERENCES}

[1] Fischer, T., Agarwal, A. \& Hess, H. A smart dust biosensor powered by kinesin motors. Nature Nanotechnology 4, 162-166 (2009).

[2] Hagleitner, C. et al. Smart single-chip gas sensor microsystem. Nature 414, 293-296 (2001).

[3] Wilson, W. C. \& Atkinson, G. M. Passive Wireless Sensor Applications for NASA's Extreme Aeronautical Environments. IEEE Sensors Journal 14, 3745-3753 (2014).

[4] Kim, J. et al. Wearable smart sensor systems integrated on soft contact lenses for wireless ocular diagnostics. Nature Communications 8, 14997, doi:10.1038/ncomms14997 (2017).

[5] Hunter, G. W., Stetter, J. R., Hesketh, P. \& Liu, C.-C. Smart sensor systems. The Electrochemical Society Interface 19, 29-34 (2010).

[6] Younis, M. I. MEMS Linear and Nonlinear Statics and Dynamics, (Springer, 2011).

[7] Yamagiwa, H. et al. Detection of Volatile Organic Compounds by Weight-Detectable Sensors coated with Metal-Organic Frameworks. Scientific Reports 4, 164 -168 (2014).

[8] Hajjaj, A. Z., Alcheikh, N., Hafiz, M. A. A., Ilyas, S. \& Younis, M. I. A scalable pressure sensor based on an electrothermally and electrostatically operated resonator. Applied Physics Letters 111, 223503, (2017).

[9] Hanay, M. S. et al. Single-protein nanomechanical mass spectrometry in real time. Nature Nanotechnology 7, 602-608 (2012). 
[10] Xu, Y., Lin, J. T., Alphenaar, B. W. \& Keynton, R. S. Viscous damping of microresonators for gas composition analysis. Applied Physics Letters $\mathbf{8 8}$, 143513, (2006).

[11] Jaber, N., Ilyas, S., Shekhah, O., Eddaoudi, M. \& Younis, M. I. Resonant Gas Sensor and Switch Operating in Air With Metal-Organic Frameworks Coating. Journal of Microelectromechanical Systems 27, 156163 (2018).

[12] Gil-Santos, E. et al. Nanomechanical mass sensing and stiffness spectrometry based on two-dimensional vibrations of resonant nanowires. Nature Nanotechnology 5, 641-645 (2010).

[13] Olcum, S., Cermak, N., Wasserman, S. C. \& Manalis, S. R. High-speed multiple-mode mass-sensing resolves dynamic nanoscale mass distributions. Nature Communications 6, 7070, (2015).

[14] Prasad, A. \& Seshia, A. A. Detection of phase transition in polyethylene glycol using a multimodal micromechanical acoustic resonator. Applied Physics Letters 110, 134101, (2017).

[15] Antonio, D., Zanette, D. H. \& López, D. Frequency stabilization in nonlinear micromechanical oscillators. Nature Communications 3, 806, (2012).

[16] Thundat, T., Wachter, E. A., Sharp, S. L. \& Warmack, R. J. Detection of mercury vapor using resonating microcantilevers. Applied Physics Letters 66, 1695-1697 (1995).

[17] Hwang, Y., Sohn, H., Phan, A., Yaghi, O. M. \& Candler, R. N. Dielectrophoresis-Assembled Zeolitic Imidazolate Framework NanoparticleCoupled Resonators for Highly Sensitive and Selective Gas Detection. Nano Letters 13, 5271-5276 (2013)

[18] Lai, Y. T. \& Yang, Y. J. A novel gas sensor using polymer dispersed liquid crystal doped with carbon nanotubes in IEEE 26th International Conference on Micro Electro Mechanical Systems (MEMS), 977-980 (2013).

[19] Henriksson, J., Villanueva, L. G. \& Brugger, J. Ultra-low power hydrogen sensing based on a palladium-coated nanomechanical beam resonator. Nanoscale 4, 5059-5064 (2012).

[20] Xu, P., Li, X., Yu, H. \& Xu, T. Advanced Nanoporous Materials for Micro-Gravimetric Sensing to Trace-Level Bio/Chemical Molecules. Sensors 14, 19023-19056 (2014).

[21] Venkatasubramanian, A. et al. MOF @ MEMS: Design optimization for high sensitivity chemical detection. Sensors and Actuators B: Chemical 168, 256-262 (2012).

[22] Shekhah, O., Liu, J., Fischer, R. A. \& Woll, C. MOF thin films: existing and future applications. Chemical Society Reviews 40, 1081-1106 (2011).

[23] Howarth, A. J. et al. Chemical, thermal and mechanical stabilities of metal-organic frameworks. Nature Reviews Materials 1, 15018 (2016).

[24] Wales, D. J. et al. Gas sensing using porous materials for automotive applications. Chemical Society Reviews 44, 4290-4321 (2015).

[25] Yassine, O. et al. H2S Sensors: Fumarate-Based fcu-MOF Thin Film Grown on a Capacitive Interdigitated Electrode. Angewandte Chemie International Edition 55, 15879-15883 (2016).

[26] Yim, C. et al. CO2-Selective Nanoporous Metal-Organic Framework Microcantilevers. Scientific Reports 5, 10674 (2015).

[27] Sapsanis, C. et al. Insights on Capacitive Interdigitated Electrodes Coated with MOF Thin Films: Humidity and VOCs Sensing as a Case Study. Sensors 15, 18153-18166 (2015).

[28] Assen, A. H., Yassine, O., Shekhah, O., Eddaoudi, M. \& Salama, K. N. MOFs for the Sensitive Detection of Ammonia: Deployment of fcu-MOF Thin Films as Effective Chemical Capacitive Sensors. ACS Sensors 2, 12941301 (2017).
[29] Xu, P., Yu, H., Guo, S. \& Li, X. Microgravimetric Thermodynamic Modeling for Optimization of Chemical Sensing Nanomaterials. Analytical Chemistry 86, 4178-4187 (2014).

[30] Lv, Y., Yu, H., Xu, P., Xu, J. \& Li, X. Metal organic framework of MOF-5 with hierarchical nanopores as micro-gravimetric sensing material for aniline detection. Sensors and Actuators B: Chemical 256, 639-647 (2017)

[31] Oprea, A. et al. Temperature, humidity and gas sensors integrated on plastic foil for low power applications. Sensors and Actuators B: Chemical 140, 227-232 (2009).

[32] Leng, X., Li, W., Luo, D. \& Wang, F. Differential Structure With Graphene Oxide for Both Humidity and Temperature Sensing. IEEE Sensors Journal 17, 4357-4364 (2017).

[33] Azzam, E. M. S. et al. Fabrication of a surface plasmon resonance biosensor based on gold nanoparticles chemisorbed onto a 1,10-decanedithiol self-assembled monolayer. Thin Solid Films 518, 387-391 (2009).

[34] Shekhah, O. et al. Post-synthetic modification of epitaxially grown, highly oriented functionalized MOF thin films. Chemical Communications 47, 11210-11212 (2011)

[35] Chernikova, V., Shekhah, O. \& Eddaoudi, M. Advanced Fabrication Method for the Preparation of MOF Thin Films: Liquid-Phase Epitaxy Approach Meets Spin Coating Method. ACS Applied Materials \& Interfaces 8, 20459-20464 (2016).

[36] Arevalo, A. et al. A versatile multi-user polyimide surface micromachinning process for MEMS applications in IEEE 10th International Conference on Nano/Micro Engineered and Molecular Systems (NEMS),561565 (2015)

[37] Bouchaala, A., Nayfeh, A. H. \& Younis, M. I. Analytical study of the frequency shifts of micro and nano clamped-clamped beam resonators due to an added mass. Meccanica 52, 333-348 (2017).

[38] Xu, P., Yu, H., Guo, S. \& Li, X. Microgravimetric Thermodynamic Modeling for Optimization of Chemical Sensing Nanomaterials. Analytical Chemistry 86, 4178-4187 (2014).

[39] Schmid, S., Villanueva, L. G. \& Roukes, M. L. Fundamentals of nanomechanical resonators. (Springer, 2016).

[40] Cleland, A. \& Roukes, M. Noise processes in nanomechanical resonators. Journal of Applied Physics 92, 2758-2769 (2002).

[41] Sansa, M. et al. Frequency fluctuations in silicon nanoresonators. Nature nanotechnology 11, 552-558 (2016).

[42] Matheny, M. H., Villanueva, L. G., Karabalin, R. B., Sader, J. E. \& Roukes, M. L. Nonlinear Mode-Coupling in Nanomechanical Systems. Nano Letters 13, 1622-1626 (2013).

[43] Westra, H. J. R., Poot, M., van der Zant, H. S. J. \& Venstra, W. J. Nonlinear Modal Interactions in Clamped-Clamped Mechanical Resonators. Physical Review Letters 105, 117205 (2010).

[44] J. Lee, W. Shen, K. Payer, T. P. Burg, and S. R. Manalis, "Toward Attogram Mass Measurements in Solution with Suspended Nanochannel Resonators," Nano Letters, vol. 10, pp. 2537-2542, 2010.

[45] A. K. Naik, M. S. Hanay, W. K. Hiebert, X. L. Feng, and M. L. Roukes, "Towards single-molecule nanomechanical mass spectrometry," Nature Nanotechnology, vol. 4, p. 445, 2009. 


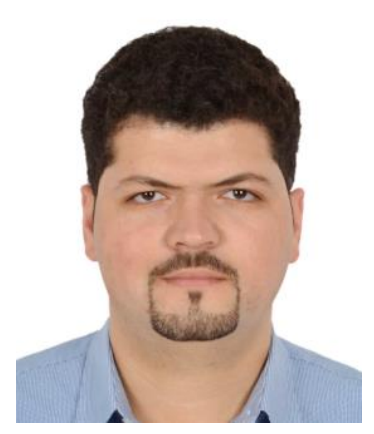

Nizar Jaber received a B.S. degree in Mechanical Engineering from Jordan University of Sciences and Technology, Jordan in 2010. He earned his master's in Mechanical Engineering from King Abdullah University of Science and Technology (KAUST) in 2014. He is currently enrolled as a $\mathrm{PhD}$ student in Mechanical Engineering in KAUST. His research interests include linear and nonlinear dynamics of MEMS based resonators with their applications in MEMS sensors and actuators.

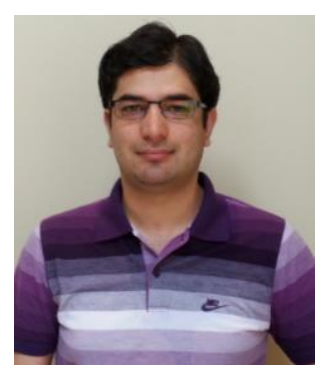

Saad Ilyas received the B.S. degree in mechanical engineering from the Ghulam Ishaq Khan Institute of Engineering Sciences and Technology, Pakistan, in 2012, and the master's degree in mechanical engineering from the King Abdullah University of Science and Technology in 2014, where he is currently pursuing the Ph.D. degree in mechanical engineering. His research interests include linear and nonlinear dynamics of MEMS devices with applications in MEMS sensors and actuators. He is also a student member of the American Society of Mechanical Engineers.

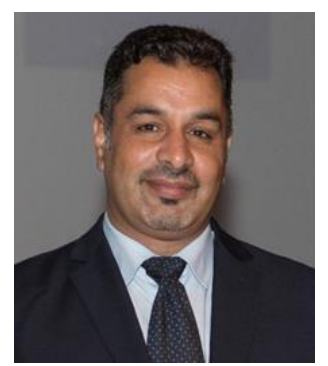

Osama Shekhah received his $\mathrm{PhD}$ in the field of physical and surface chemistry in 2004 from the Free University Berlin and Fritz-Haber Institute under the supervision of Prof. R. Schlogl. $\mathrm{He}$ then worked for one year as a postdoc at the Fritz-Haber institute in Berlin. In 2005 he joined the physical chemistry group of Prof. C. Woll at the Ruhr-University Bochum as a staff scientist. In 2009 he moved to Karlsruhe Institute of Technology with Prof. C. Woll as a group leader at the institute of functional interfaces. In 2011 he joined the FMD ${ }^{3}$ group of Prof. M. Eddaoudi at KAUST as a group leader. His research interests include surface chemistry, material chemistry, self-assembled monolayers, metal-organic frameworks (MOFs) porous material synthesis, growth and their thin films fabrication and applications.

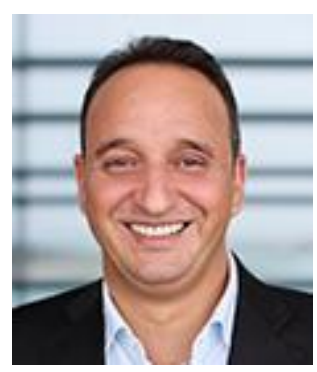

Mohammad Eddaoudi was born in Agadir, Morocco. He is currently a distinguished Professor of chemical science and the director of advanced membranes and porous materials research center, King Abdullah University of Science and Technology (KAUST, Kingdom of Saudi Arabia). He received his PhD in Chemistry, Université Denis Diderot (Paris VII), France. After postdoctoral research (Arizona State University, University of Michigan), he started his independent academic career as Assistant Professor, University of South Florida (2002), Associate Professor (2008), Professor (2010). His research focuses on developing strategies, based on (super)molecular building approaches (MBB, SBB, SBL), for rational construction of functional solid-state materials, namely MOFs. Their prospective uses include energy and environmental sustainability applications. Prof. Eddaoudi's eminent contribution to the burgeoning field of MOFs is evident from his selection in 2014 as a Thomson Reuters Highly Cited Researcher.

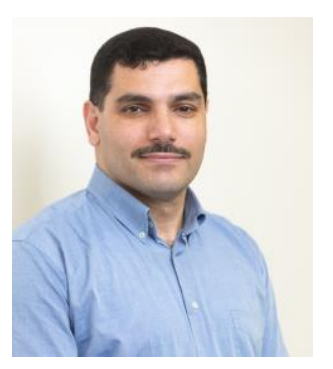

Mohammad I. Younis received the B.S. degree in mechanical engineering from the Jordan University of Science and Technology, Irbid, Jordan, in 1999, and the M.S. and Ph.D. degrees in engineering mechanics from the Virginia Polytechnic Institute and State University, Blacksburg, VA, USA, in 2001 and 2004, respectively. He is currently a Professor of Mechanical Engineering with the King Abdullah University of Science and Technology, Thuwal, Saudi Arabia, and the State University of New York (SUNY), Binghamton, NY, USA. He serves as the Director of the MEMS and NEMS Characterization and Motion Laboratory. He is a recipient of the SUNY Chancellor's Award for Excellence in Scholarship and Creative Activities in 2012, the National Science Foundation Faculty Early Career Development Award in 
2009, and the Paul E. Torgersen Graduate Research Excellence Award in 2002. He holds several U.S. patents in MEMS sensors and actuators. He serves as an Associate Editor of Nonlinear Dynamics, the Journal of Computational and Nonlinear Dynamics, and the Journal of Vibration and Control. He has authored the book MEMS Linear and Nonlinear Statics and Dynamics (Springer, 2011). He is a member of the American Society of Mechanical Engineers. 